\title{
Lepton-Flavored Scalar Dark Matter with Minimal Flavor Violation
}

\author{
Chao-Jung Lee* and Jusak Tandean ${ }^{\dagger}$ \\ Department of Physics and Center for Theoretical Sciences, National Taiwan University \\ Taipei 106, Taiwan \\ E-mail: r02222074@ntu.edu.tw, jtandean@yahoo.com
}

\begin{abstract}
We explore scalar dark matter that is part of a lepton-flavor triplet satisfying symmetry requirements under the hypothesis of minimal flavor violation. The theory contains in addition three right-handed neutrinos that participate in the seesaw mechanism for light neutrino mass generation. The dark-matter candidate couples to standard-model particles via Higgs-portal renormalizable interactions as well as to leptons through effective dimension-six operators, all of which have minimal flavor violation built-in. We consider restrictions on the triplet scalars from the Higgs boson measurements, observed relic density, dark-matter direct searches, LEP II data on $e^{+} e^{-}$ scattering into a photon plus missing energy, and searches for flavor-violating charged-lepton decays. Also, we investigate the possibility of the new scalars' interactions explaining the tentative hint of Higgs decay $h \rightarrow \mu \tau$ recently detected in the CMS experiment.
\end{abstract}

Flavor Physics \& CP Violation 2015

May 25-29, 2015

Nagoya, Japan

\footnotetext{
* Speaker.

$\dagger$ This research was supported in part by the MOE Academic Excellence Program (Grant No. 102R891505) and National Center for Theoretical Sciences of Taiwan.
} 
Despite its tremendous successes in describing particle data, the standard model (SM) cannot account for the experimental fact that neutrinos have mass [1] and has no candidate for the bulk of cosmic dark matter (DM), whose existence has been inferred from astronomical observations [1]. Among the most appealing new-physics scenarios that have been proposed to deal with these major problems are models that address them in an interconnected manner. Here we present the results of a recent investigation following a similar line of approach [2], in particular where the DM candidate carries lepton flavor and has interactions that conform to the principle of so-called minimal flavor violation (MFV), thereby linking the DM to what makes neutrinos massive.

In the MFV framework, Yukawa couplings are the only sources for the breaking of flavor symmetry [3]. Though its application to quarks is straightforward, there is no unique way to implement the notion of MFV to leptons, as the SM by itself does not predict lepton-flavor violation. Since neutrino mixing is now empirically established [1], it is attractive to formulate leptonic MFV by incorporating new ingredients in accord with that fact [4]. Thus, here we consider the SM expanded with the addition of 3 heavy right-handed Majorana neutrinos taking part in the type-I seesaw mechanism to produce light neutrino masses [5], plus a lepton-flavor triplet of complex scalars which has transformation properties adhering to the MFV criterion and contains DM of the popular weakly interacting massive particle (WIMP) type. Moreover, to ensure DM stability, we impose a $Z_{2}$ symmetry under which the new scalars are odd and other particles even.

The renormalizable mass Lagrangian for the left-handed lepton doublets $L_{k, L}$ and right-handed charged leptons $E_{l, R}$ of the $\mathrm{SM}$ and the right-handed neutrinos, $v_{l, R}$, with a mass matrix $M_{v}$ is

$$
\mathscr{L}_{\mathrm{m}}=-\left(Y_{V}\right)_{k l} \bar{L}_{k, L} v_{l, R} \tilde{H}-\left(Y_{e}\right)_{k l} \bar{L}_{k, L} E_{l, R} H-\frac{1}{2}\left(M_{v}\right)_{k l} \overline{v_{k, R}^{\mathrm{c}}} v_{l, R}+\text { H.c. },
$$

where $Y_{v, e}$ denote Yukawa coupling matrices, $H$ is the Higgs doublet, and $k, l=1,2,3$ are summed over. For $M_{v}$ eigenvalues much greater than the elements of $v Y_{v} / \sqrt{2}$, the seesaw mechanism yields light Majorana neutrinos with a mass matrix $m_{v}=-v^{2} Y_{v} M_{v}^{-1} Y_{v}^{\mathrm{T}} / 2=U_{\text {PMNS }} \hat{m}_{v} U_{\mathrm{PMNS}}^{\mathrm{T}}$ involving the Higgs's vacuum expectation value $v \simeq 246 \mathrm{GeV}$, the neutrino-mixing matrix $U_{\text {PMNS }}$, and $\hat{m}_{v}=\operatorname{diag}\left(m_{1}, m_{2}, m_{3}\right)$. This suggests $Y_{v}=i \sqrt{2} U_{\text {PMNs }} \hat{m}_{v}^{1 / 2} O M_{v}^{1 / 2} / v$ [5], where the matrix $O$ satisfies the relation $O O^{\mathrm{T}}=\mathbb{1}$. From this point on, we focus on the possibility that $O$ is real and $v_{l, R}$ are degenerate with mass $\mathscr{M}$, so that $M_{v}=\mathscr{M} \mathbb{1}$.

The MFV hypothesis $[3,4]$ then implies that $\mathscr{L}_{\mathrm{m}}$ is formally invariant under the global flavor group $G_{\ell}=\mathrm{SU}(3)_{L} \times \mathrm{O}(3)_{v} \times \mathrm{SU}(3)_{E}$. This entails that the leptons belong to the fundamental representations of their flavor groups and the Yukawa couplings are regarded as spurion fields. In other words, under $G_{\ell}$ the fields transform as $L_{L} \rightarrow V_{L} L_{L}, v_{R} \rightarrow \mathscr{O}_{v} v_{R}, E_{R} \rightarrow V_{E} E_{R}, Y_{v} \rightarrow V_{L} Y_{v} \mathscr{O}_{v}^{\mathrm{T}}$, and $Y_{e} \rightarrow V_{L} Y_{e} V_{E}^{\dagger}$, where $V_{L, E} \in \mathrm{SU}(3)_{L, E}$ and $\mathscr{O}_{v} \in \mathrm{O}(3)_{V}$ is an orthogonal real matrix.

To devise Lagrangians beyond the SM with MFV built-in, one inserts products of $Y_{v, e}$ among relevant fields to form $G_{\ell}$-invariant, SM-gauge singlet, operators [3, 4]. Special for this study are the combinations $\mathrm{A}=Y_{v} Y_{v}^{\dagger}=2 \mathscr{M} U_{\mathrm{PMNS}} \hat{m}_{v} U_{\mathrm{PMNS}}^{\dagger} / v^{2}$ and $\mathrm{B}=Y_{e} Y_{e}^{\dagger}=2 \operatorname{diag}\left(m_{e}^{2}, m_{\mu}^{2}, m_{\tau}^{2}\right) / v^{2}$. To maximize new-physics effects, we assume that $\mathscr{M}$ is large enough to make the biggest eigenvalue of $A$ equal to unity. As the $B$ elements are at most $\sim 10^{-4}$, we may then drop contributions with $B$ compared to those with $A$. It follows that, since $A^{n}$ with $n>2$ can be expressed in terms of $A$ and $A^{2}$ via the Cayley-Hamilton identity [6], our building block of interest is

$$
\Delta=\xi_{1} \mathbb{1}+\xi_{2} \mathrm{~A}+\xi_{4} \mathrm{~A}^{2}=\Delta^{\dagger},
$$


where in our model-independent approach $\xi_{1,2,4}$ are free parameters expected to be at most of $\mathscr{O}(1)$ and we have used the fact that $\operatorname{Im} \xi_{1,2,4}$ are tiny $[6,7]$.

The new scalars are complex fields which are singlet under the SM gauge group and form a triplet $\tilde{s}=\left(\begin{array}{lll}\tilde{s}_{1} & \tilde{s}_{2} & \tilde{s}_{3}\end{array}\right)^{\mathrm{T}} \sim(3,1)$ under $\mathscr{G}_{\ell}=\mathrm{SU}(3)_{L} \times \mathrm{SU}(3)_{E}$, which is the pertinent flavor group after the right-handed neutrinos are integrated out. The renormalizable Lagrangian $\mathscr{L}$ for the new scalars' interactions with each other and SM fields is then given by [2]

$$
\begin{aligned}
\mathscr{L} & =\left(\mathscr{D}^{\eta} H\right)^{\dagger} \mathscr{D}_{\eta} H+\partial^{\eta} \tilde{s}^{\dagger} \partial_{\eta} \tilde{s}-\mathscr{V}, \\
\mathscr{V} & =\mu_{H}^{2} H^{\dagger} H+\tilde{s}^{\dagger} \mu_{s}^{2} \tilde{s}+\lambda_{H}\left(H^{\dagger} H\right)^{2}+2 H^{\dagger} H \tilde{s}^{\dagger} \Delta_{H S} \tilde{s}+\left(\tilde{s}^{\dagger} \Delta_{S S} \tilde{s}\right)^{2} \\
& \supset \tilde{s}^{\dagger}\left[\mu_{s 0}^{2} \mathbb{1}+\mu_{s 1}^{2} \mathrm{~A}+\mu_{s 2}^{2} \mathrm{~A}^{2}+2 H^{\dagger} H\left(\lambda_{s 0} \mathbb{1}+\lambda_{s 1} \mathrm{~A}+\lambda_{s 2} \mathrm{~A}^{2}\right)\right] \tilde{s}+\left[\tilde{s}^{\dagger}\left(\lambda_{s 0}^{\prime} \mathbb{1}+\lambda_{s 1}^{\prime} \mathrm{A}+\lambda_{s 2}^{\prime} \mathrm{A}^{2}\right) \tilde{s}\right]^{2},
\end{aligned}
$$

where $\mu_{s j}^{2}, \lambda_{s j}$, and $\lambda_{s j}^{\prime}$ are free real parameters and further details can be found in [2]. Due to the Hermiticity of A, the matrices sandwiched between $\tilde{s}^{\dagger}$ and $\tilde{s}$ in (4) can be simultaneously diagonalized, and so $\tilde{s}_{k}$ are related to the mass eigenstates $S_{k}$ by $S=\left(\begin{array}{lll}S_{1} & S_{2} & S_{3}\end{array}\right)^{\mathrm{T}}=\mathscr{U}^{\dagger} \tilde{s}$, with $\mathscr{U}=U_{\mathrm{PMNS}}$ being the unitary matrix that diagonalizes $A$.

In the MFV framework, $S_{1,2,3}$ can couple to SM leptons via effective dimension-6 operators:

$$
\begin{array}{rlrl}
\mathscr{L}^{\prime} & =\frac{C_{b d k l}^{L}}{\Lambda^{2}} O_{b d k l}^{L}+\frac{C_{b d k l}^{R}}{\Lambda^{2}} O_{b d k l}^{R}+\left(\frac{C_{b d k l}^{L R}}{\Lambda^{2}} O_{b d k l}^{L R}+\text { H.c. }\right), & \\
C_{b d k l}^{L} & =\left(\Delta_{L L}\right)_{b d}\left(\Delta_{S S}\right)_{k l}+\left(\Delta_{L S}\right)_{b l}\left(\Delta_{s L}\right)_{k d}+\left(\Delta_{L S}\right)_{k d}\left(\Delta_{S L}\right)_{b l}, & & O_{b d k l}^{L}=i \bar{L}_{b, L} \gamma^{\rho} L_{d, L} \tilde{s}_{k}^{*} \stackrel{\leftrightarrow}{\partial_{\rho}} \tilde{s}_{l}, \\
C_{b d k l}^{R} & =\delta_{b d}\left(\Delta_{S S}^{\prime}\right)_{k l}, & & O_{b d k l}^{R}=i \bar{E}_{b, R} \gamma^{\rho} E_{d, R} \tilde{s}_{k}^{*} \vec{\partial}_{\rho} \tilde{s}_{l}, \\
C_{b d k l}^{L R} & =\left(\Delta_{L Y} Y_{e}\right)_{b d}\left(\Delta_{S S}^{\prime \prime}\right)_{k l}+\left(\Delta_{L S}^{\prime}\right)_{b l}\left(\Delta_{S Y} Y_{e}\right)_{k d}, & & O_{b d k l}^{L R}=\bar{L}_{b, L} E_{d, R} \tilde{s}_{k}^{*} \tilde{s}_{l} H,
\end{array}
$$

where summation over $b, d, k, l=1,2,3$ is implicit, the mass scale $\Lambda$ characterizes the underlying heavy new-physics, and the $Z_{2}$ symmetry has been imposed. The $\Delta$ 's in $C^{L, R, L R}$ above are of the form in (2), but have generally different coefficients $\xi$ 's.

We can take $S_{3}$ to be sufficiently lighter than $S_{1,2}$ and serve as a good DM candidate [2]. In that case, $\lambda_{3} \equiv \lambda_{s 0}+2 \lambda_{s 1} \mathscr{M} m_{3} / v^{2}+4 \lambda_{s 2} \mathscr{M}^{2} m_{3}^{2} / v^{4}$, from (4), controls the Higgs-mediated annihilations of the DM into SM particles, its scattering off a nucleon via Higgs exchange, and the Higgs nonstandard invisible decay if the $S_{3}$ mass is less than half of the Higgs mass, $m_{S_{3}}<m_{h} / 2$. Some of these processes also receive contributions from the dimension-6 operators in $\mathscr{L}^{\prime}$. Thus the new interactions are subject to constraints from the pertinent existing data.

We entertain first the possibility that the dimension- 6 operators are absent. Accordingly, with the parameter selections and other formulas described in [2], we can extract the $\left|\lambda_{3}\right|$ values consistent with the observed relic density [1] and compare them to the upper limits on $\left|\lambda_{3}\right|$ inferred from the null result of WIMP DM direct search by LUX [8] and from the condition $\mathscr{B}\left(h \rightarrow S^{*} S\right)<0.19$ based on new-physics bounds from Higgs measurements [9]. We display the results in Figure 1. It indicates that the $\lambda_{3}$ contribution to the DM annihilation rate is much less than half of the necessary amount if $m_{S_{3}}<90 \mathrm{GeV}$, except near $m_{S_{3}}=m_{h} / 2$. Thus, over most of this mass region the dominant contribution would have to come from the effective interactions. For larger $m_{S_{3}}$, on the other hand, each of the two sources can yield a nonnegligible effect on the relic density.

Next, we examine a scenario in which $\lambda_{3}$ vanishes and the effective operators in (5) are responsible for the relic abundance. To illustrate the potential implications, we make the simple choices 


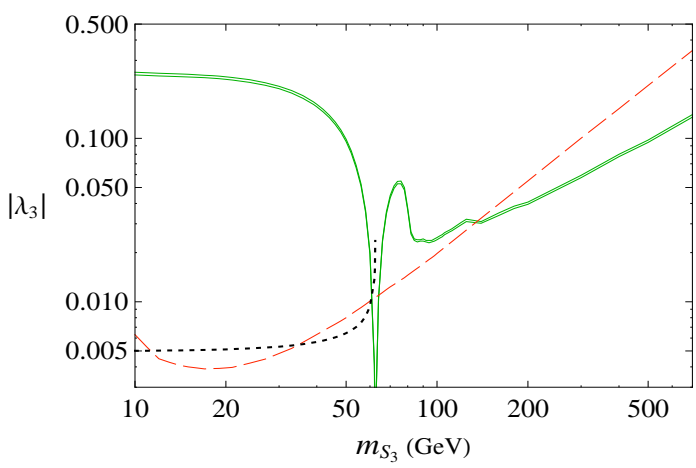

Figure 1: The values of $\left|\lambda_{3}\right|$ versus $S_{3}$ mass consistent with the relic density data (green solid curve), compared to the upper limits on $\left|\lambda_{3}\right|$ from Higgs measurements (black dotted curve) and WIMP DM direct searches (red dashed curve), in the absence of the dimension-6 operators in $\mathscr{L}^{\prime}$.

$C_{b d k l}^{L R}=\sqrt{2} \delta_{b l} \delta_{d k} \kappa_{L R} m_{\ell_{d}} / v, C_{b d k l}^{L}=2 \delta_{b l} \delta_{d k} \kappa_{L}$, and $C_{b d k l}^{R}=\delta_{b d} \delta_{k l} \kappa_{R}$ with $\kappa_{L R, L, R}$ being real constants. Assuming that only one of $\kappa_{L R, L, R}$ is nonzero at a time, we then arrive at the results depicted in the left plot of Figure 2. In these cases, constraints from DM direct searches are unimportant.

There are complementary restrictions on the effective lepton- $S$ couplings which are consequential. Since they can contribute to the monophoton production process $e^{+} e^{-} \rightarrow \gamma \notin$ with missing energy $\mathbb{E}$, we can probe them further with the available data on this reaction from LEP II, tabulated in [10]. Also relevant are the experimental limits [1] on the flavor-changing charged-lepton decays $\ell_{a}^{-} \rightarrow \ell_{b}^{-} \ell_{c}^{-} \ell_{d}^{+}$which get one-loop contributions from the couplings. This extra experimental information allows us to improve the constraints on the couplings significantly, as illustrated in the right plot of Figure 2 for the $\varepsilon=L$ case. The dotted green curve suggests that a next-generation $e^{+} e^{-}$ collider with higher energy and luminosity can offer more stringent tests on them.

Now, if the Higgs interactions from (4) and effective couplings in (6) are simultaneously present, they may induce lepton-flavor-violating Higgs decays. This is of great interest because
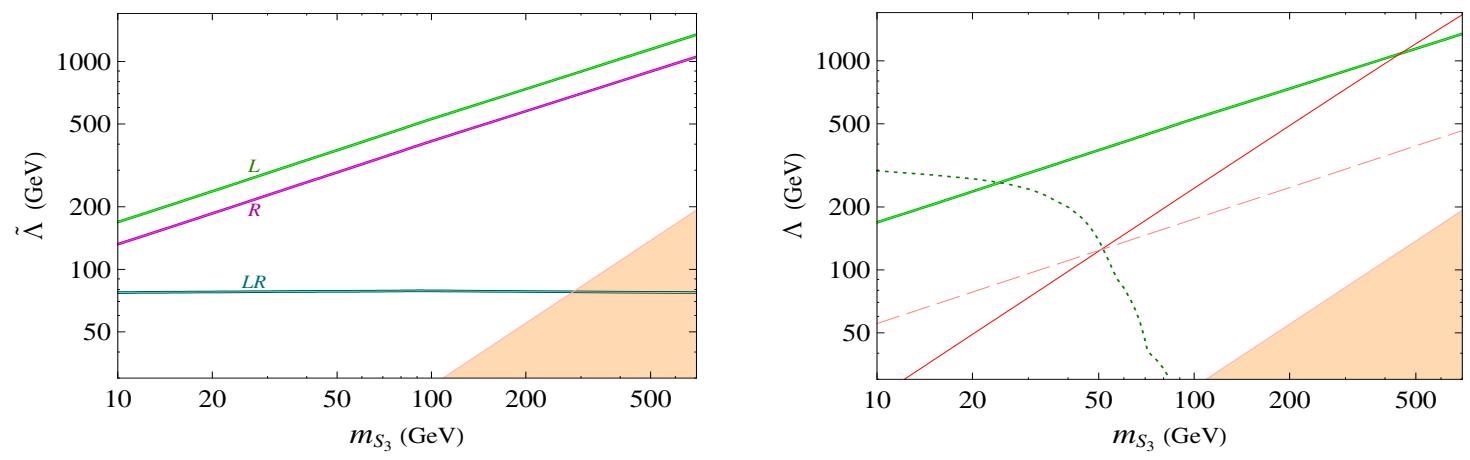

Figure 2: Left: the values of $\tilde{\Lambda} \equiv \Lambda /\left|\kappa_{\varepsilon}\right|^{1 / 2}$ versus $S_{3}$ mass for $\varepsilon=L, R, L R$ fulfilling the relic density requirement, in the absence of the Higgs-mediated $\lambda_{3}$ contributions. Right: the $\Lambda$ values from the $\varepsilon=L$ example in the left plot with $\kappa_{L}=1$, compared to the lower limits on $\Lambda$ from the LEP II data on $e^{+} e^{-} \rightarrow \gamma E$ (green dotted curve) and from searches for $\mu^{-} \rightarrow e^{-} e^{-} e^{+}$(red solid curve) and $\tau^{-} \rightarrow \mu^{-} \mu^{-} e^{+}$(red dashed curve). In each of the graphs, the (orange) shaded region depicts the parameter space where the effective field theory approach breaks down. 
the CMS Collaboration [11] has recently reported the detection of a slight excess of $h \rightarrow \mu^{ \pm} \tau^{\mp}$ events with a significance of $2.5 \sigma$. If interpreted as a signal, the result implies a branching fraction of $\mathscr{B}(h \rightarrow \mu \tau)=\mathscr{B}\left(h \rightarrow \mu^{-} \tau^{+}\right)+\mathscr{B}\left(h \rightarrow \mu^{+} \tau^{-}\right)=\left(0.89_{-0.37}^{+0.40}\right) \%$, but as a statistical fluctuation it corresponds to the limit $\mathscr{B}(h \rightarrow \mu \tau)<1.57 \%$ at $95 \%$ confidence level [11]. It is too soon to draw a definite conclusion from this finding, but it would be evidence of new physics if confirmed by future experiments. Thus it is germane to explore if our triplet scalars' interactions can accommodate the hypothetical signal from CMS. Indeed, as detailed in [2], taking into account the latest LHC data on $h \rightarrow \mu^{+} \mu^{-}, \tau^{+} \tau^{-}$[12] and adopting the above choice of the $\varepsilon=L R$ effective coupling, we obtain $0.52 \%<\mathscr{B}(h \rightarrow \mu \tau)<0.79 \%$, which is within the positive result range.

In conclusion, we have considered scalar DM which is a member of a lepton-flavor triplet and stabilized by a $Z_{2}$ symmetry. We apply the MFV principle to all the lepton-flavored particles in the theory, including the right-handed neutrinos responsible for the seesaw mechanism to create light neutrino masses. The triplet scalars couple to SM particles via Higgs-portal renormalizable interactions and effective dimension-6 operators involving leptons. The MFV framework allows us to develop interesting phenomenological links among the DM, Higgs, and lepton sectors. We also look at whether the new scalars' interactions can explain the tentative hint of Higgs decay $h \rightarrow \mu \tau$ recently seen by CMS, finding that they can produce a rate compatible with the CMS result and at the same time be consistent with other Higgs data. Lastly, it is clear from our study that future $e^{+} e^{-}$machines with high energies and luminosities, such as the International Linear Collider, have a lot of potential for testing in improved detail different aspects of this kind of MFV scenario, such as the couplings of the new scalars to the Higgs boson and to leptons.

\section{References}

[1] K.A. Olive et al. [Particle Data Group Collaboration], Chin. Phys. C 38, 090001 (2014).

[2] C.J. Lee and J. Tandean, JHEP 1504, 174 (2015).

[3] G. D’Ambrosio, G.F. Giudice, G. Isidori, and A. Strumia, Nucl. Phys. B 645, 155 (2002).

[4] V. Cirigliano, B. Grinstein, G. Isidori, and M.B. Wise, Nucl. Phys. B 728, 121 (2005).

[5] M. Raidal et al., Eur. Phys. J. C 57, 13 (2008).

[6] L. Mercolli and C. Smith, Nucl. Phys. B 817, 1 (2009).

[7] X.G. He, C.J. Lee, S.F. Li, and J. Tandean, Phys. Rev. D 89, 091901 (2014); JHEP 1408, 019 (2014).

[8] D.S. Akerib et al. [LUX Collaboration], Phys. Rev. Lett. 112, 091303 (2014).

[9] K. Cheung, J.S. Lee, and P.Y. Tseng, Phys. Rev. D 90, 095009 (2014).

[10] C.W. Chiang, G. Faisel, Y.F. Lin, and J. Tandean, JHEP 1310, 150 (2013).

[11] CMS Collaboration, Report No. CMS-PAS-HIG-14-005, July 2014.

[12] CMS Collaboration, Report No. CMS-PAS-HIG-14-009; ATLAS collaboration, Report No. ATLASCONF-2014-061, ATLAS-COM-CONF-2014-080; G. Aad et al. [ATLAS Collaboration], Phys. Lett. B 738, 68 (2014); V. Khachatryan et al. [CMS Collaboration], Phys. Lett. B 744, 184 (2015). 